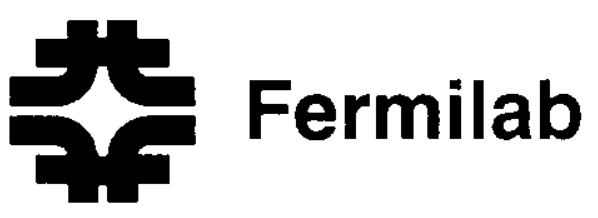

TM-1405

0400.000

RESONANCES AND RESONANCE WIDTHS

T. Collins

May 1986 


\title{
Resonances and Resonance Widths
}

\author{
Tom Collins
}

Fermilab, May 1986

Two-dimensional betatron resonances are much more important than their simple one-dimensional counterparts and exhibit a strong depdndencos on the betatron phase advance per cell. This paper enlarges upon a note, of limited distribution, which used a practical definition of "width" in order to display these relations in tables.

A substantial introduction has been added to this note, primarily pedagogical, to explain the tables, and also to encourage a wider capability for deriving resonance behavior and wider use of "designer" resonances. 
Many years ago Don Edwards and I in a fundamental design paper for the Doubler argued strenuously against a "cost-saving" reduction of the phase advance per cell. To do so we used a table of widths for two-dimensional resosnances and pointed to the strong dependence on phase advance. Three years ago, following the first Snowmass Summer Study, I enlarged this table and refined the phase dependence in a small note of limited distribution, because of proposed "great cost-savings". Following the second snowmass Summer Study, I shifted to non-resonant distortion and "smearing" criteria (same purpose) in Distortion Functions, which is a complementary approach to resonance theory with a direct numerical relation to performance quality but which also contains much of interest for resonances.

What remains from these efforts, apart from an excellent Doubler, is a recurring interest in the table of widths and I have been encouraged to insert a short explanation of the numerical methods into the small note and give it a wider distribution.

Resonance theory is a lot of fun, so I could not resist meandering along a few of the many fascinating by-ways before explaining the last three handdrawn pages which were the bulk of the small note.

\section{Resonance Width}

The terms resonance and resonance width are commonly used in physics, usually without explanation, but they have a special meaning for betatron tune resonances in accelerator rings for which there is no analgous behaviour in electrical circuitry. One betatron phenomenon does resemble a normal resonance - the growth of orbit distortion as the betatron tune approaches an integer. In this case there is a denominator $(\sin \pi \nu)$ which approaches zero and in electrical terminology this is a very narrow resonance (measured down from the peak), but an accelerator operator would find it very wide. Note that for any small tune there is a stable beam, but not a useful one, and the tune itself is unmodified. We now contrast this behaviour with "real" resonances.

First we must distinguish clearly the tune from the tuning. Tune is the betatron phase advance per turn, measured in revolutions where the fractional part is the significant quantity. There are two tunes, $\nu_{x}, \nu_{y}$, which can be measured directly from the beam. Tune varies somewhat with the amplitudes and with momentum. Tuning is the setting of tuning quadrupoles, converted to tune units by simple formulas. For a well behaved, small beam with no momentum error, the tunes follow the tunings. 
Consider then tuning towards the half-integer. When just below one finds that the tune runs ahead of the tuning and reaches $1 / 2$ when the tuning is slightly less. Coming down from above one again finds the tune running ahead to $1 / 2$ but this time the tuning is slightly greater than $1 / 2$. For a tuning between these limits there is no stable beam. In this region the beam is represented by a tune of $1 / 2$ multiplied by an exponential growth in amplitude (a complex phase angle). We use the tuning width of the region where the tune is locked to $1 / 2$ as a measure of the resonance strength. Note that there are no missing tunes, they are just a little crowded on each side of the locked region.

Now let there be a scatter of sextupoles in the ring. We will find a locked region at $\nu_{x}=1 / 3$, but in this case the tuning width is proportional to the amplitude. (If one uses 10-poles it would be $a \mathrm{mp}^{3}$.) One way to quote a single tuning width for each resonance is to use a standard amplitude (1cm), however if we set $\nu_{x}$ near $1 / 3$ and vary $\nu_{y}$ we will find another, larger resonance when $\nu_{x}+2 \nu_{y}=$ integer.

On a tuning-plot, where $3 \nu_{x}$ is a vertical line, this two-dimensional resonance is a narrow band through $(1 / 3,1 / 3)$ with a slope of $-2 / 3$. [Identification of resonances requires a line on a tuning plot, if you can't find a line then it isn't a resonance.] The tuning width for this resonance not only depends on the general level of amplitudes but also in a complex way on the specific mix of horizontal and vertical amplitude. We need a practical definition of width in order to compare two-dimensional resonance strength.

For "resonance width" I use the tuning width for beam loss, measured perpendicular to the resonance line, between boundaries where a gaussian beam - with equal $\sigma$ in both planes - loses $10 \%$. This corresponds closely to the value that an accelerator operator would quote, and is an adequate measure for assessing the difficulties of avoiding beam loss.

Betatron oscillations can be observed by a frequency analysis of position monitor signals, where they appear as side-bands to the orbital frequency. One is tempted to continue to think in terms of frequencies using electrical analogies for assistance, however there is no electrical counterpart to this particular non-linear problem. The locking above is not related in any way to the "pulling" of an oscillator - the pulled oscillator keeps on oscillating, and it's the linear oscillator that has no stable amplitude. The analysis of betatron resonances is best carried out in a time domain, that is turn-byturn, and the physics of the process is particularly explicit using an amplitude-phase description of the oscillations. 
Preliminaries

We will examine resonances in the tunes $m \nu_{x}+n \nu_{y}=$ integer, $m+n=k$. These "locked" resonances are narrow bands on a tuning diagram ( $\nu_{x}$ vs $\left.\nu_{y}\right)$ with a tuning width $\delta( \pm \delta / 2)$ measured perpendicular to the band. You will find easily that the tuning relation for the band edges is

$$
m \nu_{x}+n \nu_{y}=\text { integer } \pm\left(m^{2}+n^{2}\right)^{1 / 2}(\delta / 2) .
$$

A phase $\varphi$ is associated with $\nu_{x}$, and $\vartheta$ with $\nu_{y}$. It is convenient to define $\alpha=m \varphi+n \theta$, and $\delta_{\alpha}=\left(m^{2}+n^{2}\right)^{1 / 2} \pi \delta$ as the extra $\alpha$ per turn.

At the "locking" boundaries $\delta_{\alpha}$ is, on the average, cancelled by additional phase from non-linear elements (multipoles).

One can write the multipole fields as

$$
\begin{gathered}
B_{y}=B \sum\left[b_{k-1} f(X, Y)-a_{k-1} g(X, Y)\right] \\
B_{x}=B \sum\left[a_{k-1} g(X, Y)+b_{k-1} f(X, Y)\right] \\
\text { (note: } k=3 \rightarrow b_{2} \rightarrow \text { sextupole } \rightarrow 2 k \text {-pole) }
\end{gathered}
$$

\begin{tabular}{l:ll}
$k$ & $f(X, Y)$ & $g(X, Y)$ \\
\hdashline $2:$ & $X$ & $Y$ \\
3 & $X^{2}-Y^{2}$ & $2 X Y$ \\
4 & $X^{3}-3 X Y^{2}$ & $3 X^{2} Y-Y^{3}$ \\
5 & $X^{4}-6 X^{2} Y^{2}+Y^{4}$ & $4 X^{3} Y-4 X Y^{3}$ \\
$k:$ & $(-1)^{n} / 2\left(k_{n}\right)(m / k) X^{m-1} Y^{n}$ & $-(-1) n / 2\left(k_{n}\right)(n / k) X^{m} Y^{n-1}$ \\
& for $n=0,2,4 \ldots$ and $m+n=k$, & $\left(k_{m}\right)=k ! /(m ! n !)=\left(k_{n}\right)$
\end{tabular}

In the generalized terms one is anticipating that $m, n$ will become the same as used in the resonance expression, otherwise the terms could be simpler.

We will want to define displacements as

$$
\begin{array}{rlrl}
x & =a \cos \varphi, & y & =b \cos \vartheta, \quad \text { measured at } \beta_{0} \\
(d x / d \varphi)=x^{\prime} & =-a \sin \varphi, & (d y / d \vartheta)=y^{\prime}=-b \sin \vartheta . & \\
x & =\left(\beta_{x} / \beta_{0}\right)^{1 / 2} X, \quad x^{\prime}=\left(\beta_{x} \beta_{0}\right)^{1 / 2} X^{\prime}+\left(\beta^{\prime} / 2\right)_{X} & \text { etc. }
\end{array}
$$

It will be convenient to use $h=\left(\beta_{x} / \beta_{0}\right)^{1 / 2}$ and $v=\left(\beta_{y} / \beta_{0}\right)^{1 / 2}$.

A kick $\Delta X^{\prime}$ at $\beta_{x}$ must be converted to $\Delta X^{\prime}=\left(\beta_{x} \beta_{0}\right)^{1 / 2} \Delta X^{\prime}$

$$
\begin{aligned}
& \Delta x^{\prime}=h \beta_{0} \Delta X^{\prime}=-h \beta_{0}\left(B_{y} l /\left[B_{\rho}\right]\right), \text { for a short length } l \\
& \Delta y^{\prime}=v \beta_{0} \Delta Y^{\prime}=v \beta_{0}\left(B_{x} I /\left[B_{\rho}\right]\right) .
\end{aligned}
$$


We will want to continue to use phase-amplitude expressions because tune-shifts appear explicitly, so convert a small $\Delta x^{\prime}$ or $\Delta y^{\prime}$ to

$$
\begin{array}{ll}
\Delta \varphi=-\left(\Delta x^{\prime} / a\right) \cos \varphi, & \Delta \vartheta=-\left(\Delta x^{\prime} / b\right) \cos \vartheta, \\
\Delta a=-\Delta y^{\prime} \sin \varphi, & \Delta b=-\Delta y^{\prime} \sin \vartheta .
\end{array}
$$

For example at $x_{\max }(\varphi=0)$, a small kick moves the phase back to a positive slope without significant change in $a=\left(x^{2}+x^{\prime 2}\right)^{1 / 2}$.

Resonance Algebra

We must make a fundamental assumption that beam behaviour when not resonant can be well described by simple linear theory, that is the phaseamplitude equations above for $x$ and $y$ are meaningful. In this case the deviations caused by non-linear fields in any one turn are small, and in general remain small after many turns. Exceptional cases are created by components of the field which are resonant or which generate amplitude dependent tuneshifts, and in these cases small deviations accumulate for many turns.

In the analysis below we ignore the unexceptional terms. This is necessary to obtain a solution - a simple expression predicting beam behavior - and it is also a reasonable approximation. It is also necessary to avoid resonancecrossings, tune combinations which simultaneously satisfy more than one set of $m$ and $n$. There is no egregious beam behavior at crossing resonances but it is too complex for solution. This particular method of analysis is simple and directly related to numerical evaluation, nevertheless the limitations and conclusions are identical to more elegant methods.

We first examine a simple one-dimensional resonance and then we use the generalized field terms to develop solutions for the principle two-dimensional resonances which are more important. The simple example is contained in the more general solution, and in its development we will take "simplifying" steps that could easily be omitted but actually mimic the two-dimensional case.

An octupole field $B_{y}=B b_{3} X^{3}$ with length $l$ produces

$$
\begin{aligned}
\Delta x^{\prime} & =-\left(\beta_{0} h\right)\left(B_{0} l /[B \rho]\right) b_{3}(h a \cos \varphi)^{3} l \\
\Delta \varphi & =\left(\beta_{0} B /[B \rho]\right) a^{2}\left(h^{4} b_{3} l \cos ^{4} \varphi\right) \\
\Delta a & =\left(\beta_{0} B /\left[B_{\rho}\right]\right) a^{3}\left(h^{4} b_{3} l \cos ^{3} \varphi \sin \varphi\right)
\end{aligned}
$$

$\cos ^{4} \varphi=(1 / 8)[\cos 4 \varphi+4 \cos 2 \varphi+3], \cos ^{3} \varphi \sin \varphi=(1 / 8)[\sin 4 \varphi+2 \sin 2 \varphi]$

We will make the $4 \varphi$ term resonant by choosing $\nu_{x}=$ integer $+1 / 4+\delta / 2$. The $2 \varphi$ terms will be ignored, and the tune-shift contribution ( $3 / 8$ in $\Delta \varphi)$ will be considered later. 
We are going to examine the effects of the $4 \varphi$ component of an array of octupole fields by observing beam behaviour at one point in the ring for sucessive turns. We will now use $a, \varphi$ for values at the start of a turn, and use $\varphi+\varphi_{s}$ for the particle phase when it reaches a particular element. After one turn $\Delta a$ will be the simple sum of contributions from all the elements. For one turn $\Delta \varphi$ will also include the normal phase advance from the tuning.

Let me use $\alpha$ for $4 \varphi, \alpha_{s}$ for $4 \varphi_{s}, \delta_{\alpha}$ for $4 \pi \delta$. We can express the $\Delta^{\prime} s$ in terms of starting values and the following sums:

$$
A=\left(\beta_{0} B /[B \rho]\right) \sum\left(h^{4} b_{3} l\right)_{s} \cos \alpha_{s}, \text { and } B=(. .) \sum\left(h^{4} b_{3} l\right)_{s} \sin \alpha_{s}
$$

For one turn, at resonance, $\alpha_{s} / 2 \pi$ is an integer so $A$ and $B$ are essentially Fourier components and the combined amplitude $\left(A^{2}+B^{2}\right)^{1 / 2}$ is independent of the choice of observation point. We can simplify our formulas uy choosing an observation point where $B$ is zero and $A$ is a maximum (positive), then

$$
\begin{aligned}
\Delta \varphi & =(1 / 8) A a^{2} \cos \alpha+90^{\circ}+\pi \delta / 2 \text { per turn } \\
\text { or } \quad \Delta \alpha & =(1 / 2) A a^{2} \cos \alpha+\delta_{\alpha} \\
\text { and } \Delta a & =(1 / 8) A a^{3} \sin \alpha
\end{aligned}
$$

This is a good time for comments on the derivation and evaluation of the driving term $A$.

We are assuming that non-linear "kicks" are small, and when adding the effect of an element to the one-turn sums we ignore previous non-linear kicks on the same turn when we calculate $X$ for use in $b_{3} X^{3}$. This is called a firstorder solution, and it is generally sufficient for random errors. A secondorder solution can be developed (conceptually) by adding at $s$ terms like $3 b_{3} X^{2} \Delta X$ to a new set of second-order sums, where $\Delta X$ is the first-order effect of multipoles between the starting point and $s$. Now $\Delta X$ already contains $a$ complex dependence on $\varphi+\varphi_{s}$, so the trigonometric terms will become more complex and their expansion is a new set of second-order resonances.

One can expect all sum and difference combinations of the first-order expansions of the combined multipoles. For example, sextupoles have first-order terms $\varphi$ and $3 \varphi$, and second-order expansions $0,2 \varphi, 4 \varphi,(6 \varphi)$ (the driving term for $6 \varphi$ is always zero). A combination of sextupoles and octupoles will have $\varphi$, $3 \varphi \ldots . .7 \varphi$. Significant resonances from these combinations are usually from systematic arrays, such as a few strong correction elements arranged to make undesirable first-order driving terms zero, but not higher orders or interactions with other corrections. 
It is important when computing a systematic driving term to us the real values for $\beta$ and $\varphi$ that describe the ring when it is tuned to the resonance frequency by the actual tuning quadrupoles. One often writes $\varphi=\nu \psi$, $\psi=0 \rightarrow 2 \pi$ but then assumes that tuning changes $\nu$ but not $\psi$. This is never correct for a real lattice with straight sections. In the Doubler the tuning is confined to the arcs, which minimizes $\beta$ changes, and the phase advances of the straight sections are almost unaffected. For very large rings it is probable that tuning will be confined to tuning cells in the straight sections. One must also be aware that "turning on" low beta straight sections (and restoring the tune) may drastically affect all driving terms.

A last comment on driving terms - this is the time to transform $A$ to sensible units. In the expression $A=\left(\beta_{0} B /[B \rho]\right) \sum\left(h^{k} b_{k-1} l\right)_{s} \cos k \varphi_{B}$ the first term will normally use tesla-m in both numerator and denominator so the units

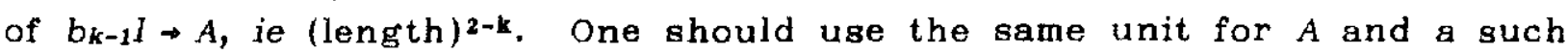
that the amplitude is close to unity, such as $\mathrm{cm}$. or $\mathrm{mm}$. If one persists in using meters then higher multipole driving terms will have huge values even if they are negligible. For a dipole one can write $A=\beta_{0} \sum\left(h^{k} b_{k-1} \theta\right)_{s} \cos k \varphi_{s}$ where $\theta$ is the bend angle and in this case it is units $\beta_{0} b_{k-1} \rightarrow A$.

We return to the simple $4 \varphi$ resonance, and having just found reasonable units for $a$, we promptly make an unecessary simplification and write $a$ in terms of a new scaling unit ao, where $a_{0}=\left\{8 \delta_{\alpha} / A_{1}^{1 / 2}\right.$ (without explanation, all will be clear for two dimensions). Let me summarize:

$$
\begin{gathered}
\nu_{x}=\text { integer }+1 / 4+\delta / 2 \\
\alpha=4 \varphi \quad \delta_{\alpha}=4 \pi \delta \\
A=\text { maximum of }\left(\beta_{0} B /[B \rho]\right) \sum\left(h^{4} b_{3}\right)_{s} \cos \alpha_{s} \\
a_{0}=i 8 \delta_{\alpha} / A_{i}^{1 / 2} \quad u=a / a_{0} \\
d u / d N=\delta_{\alpha} u^{3} \sin \alpha \\
d \alpha / d N=\delta_{\alpha}\left(4 u^{2} \cos \alpha \pm 1\right) \quad N \text { is turns }
\end{gathered}
$$

Locking is now quite explicit. There is no change in $u$ or $\alpha$, called fixed points, when $u=.5$ and, for $\alpha=180^{\circ}(\delta / 2$ pos), or $\alpha=0(\delta / 2$ neg $)$. In both cases $\varphi$ increases by exactly $90^{\circ}$ per turn (tune $=1 / 4$ ) but the tuning differs by $\delta / 2$.

I now invite you to verify by differentiation, first by $u$ and then by $\alpha$, and using the differentials above, that for any particle

$$
2 u^{4} \cos \alpha+u^{2}=\text { constant }
$$


This kind of expression is known as a constant of the motion, for our purposes however it is the trajectory in phase space which a particle will follow. It can be easily converted to lines on a $x / a_{0}$ vs $x^{\prime} / a_{0}$ diagram. Solving a resonance problem means finding a constant of the motion, and many problems apparently do not have a simple algebraic solution. All solutions in this paper have been found by the good-guess-and-test process (as in integration).

The most significant trajectory is the one containing the fixed-points. For positive $\delta$ one inserts $u=.5$ and $\cos \alpha=-1$ to find

$$
2 u^{4} \cos \alpha+u^{2}=1 / 8
$$

which is known as the equation of the separatrix. In figure 1 this equation is plotted as solid lines on an $x / a_{0}, x^{\prime} / a_{0}$ diagram hyperbolas with fixed-points at the intersections.

Dotted lines represent other trajectories, with the dots indicating turns starting from the $x$-axis. For each value of $\cos \alpha$ there are four values for $\varphi$, and hence a four-fold symmetry with successive turns moving from quadrant to quadrant clockwise.

All outside particles (const.>1/8) are swept out along one of the four-fold separatrices and are lost. Particles inside $(<1 / 8)$ are stable. The $\cos \alpha$ term is

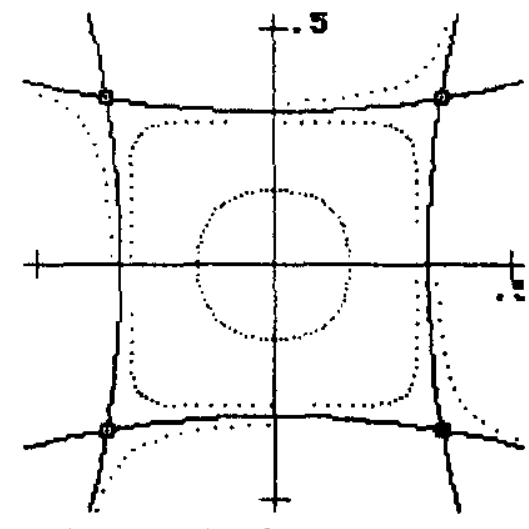

fig. 1 1/4's resonance not significant for a small constant, and the equation reduces to its normal form $a^{2}=$ const., as seen in the inner trajectory.

Consider how a fixed-point particle moves on the phase diagram as one follows it around the ring. As usual it will rotate clockwise many times but in this case the net phase advance after one turn is exactly $90^{\circ}$, precisely closing in four turns. The whole phase diagram rotates with the fixed-point in a Moebius rope which seems to be woven from four strands but actually is only one. When we chose the observation point so as to eliminate $B$ we simply chose a particular orientation of the diagram (with simpler formulas).

The strength of the resonance is given by the driving term $A$. To convert this to a tuning width it is necessary to multiply $A$ by some amplitude squared. The usual choice is the fixed-point amplitude, thus

$$
u^{2}=.25 \rightarrow a^{2}=.25(32 \pi \delta / A) \text { or } \delta=.125 a^{2} A / \pi
$$

and to arbritrarily set the fixed point amplitude a to $1 \mathrm{~cm}$. This definition ignores the normal tuning process and is not useful for two-dimensional resonances. 
A better choice for the $(\mathrm{amp})^{2}$ is $\vec{u}^{2}$, averaged over $\alpha$, which when multiplied by $\pi$ is the area inside the separatrix, thus for our case

$$
\vec{u}^{2}=.1043 \rightarrow \vec{a}^{2}=.1043(32 \pi \delta / A\rangle \text { or } \delta=.223\langle a\rangle^{2} A / \pi
$$

Consider a disk of particles in phase space with maximum amplitude 〈a〉 and a uniform density, and at a tune well above resonance - the amplitude for the fixed-points is at least several times $\langle a\rangle$. On tuning slowly down to the resonance, the separatrix squeezes down onto the beam, distorting the shape, and at $\delta / 2$ the beam will fill the separatrix (it keeps the same uniform density, that's Liouvilles theorem). Further tuning causes beam loss. If we start below the resonance and tune up, the same conditon is found at $-\delta / 2$.

This $\delta$ is an adiabatic tuning width for a remote amplitude $\langle a\rangle$, and it is conceptually more satisfying because the tuning process, which is essential to any definition, is explicit. Let me extend this further by assuming that the remote beam has a gaussian density distribution given by $\sigma$ measured at $\beta_{0}$. I now choose the amplitude containing $90 \%$ of the beam as $\langle a\rangle$, in other words I define the tuning width as $\delta$ for $10 \%$ loss points, then

$$
\langle a\rangle^{2}=4.605 \sigma^{2} \text { and } \delta=1.026 \sigma^{2} \mathrm{~A} / \pi \text {. }
$$

This definition, with some numerical effort, can be used for all resonances.

\section{Closed Resonances}

The resonance we have been considering is called open because the arms of the separatrix extend beyond the vacuum chamber (infinity). Most resonances however are closed and the arms are joined. This is caused by the inevitable dependence of tune on amplitude. In general

$$
\Delta \nu x=f\left(a^{2}, b^{2}, a^{4}, a^{2} b^{2}, b^{4} \ldots \ldots\right)
$$

and, yes, $\nu_{x}$ does depend on the $y$ amplitude. The coefficients in this polynomial have either sign, are not particularly correlated, and come from many sources - including beam-beam interaction.

As an example we continue with the same $4 \varphi$ resonance but add a term

$$
\begin{aligned}
& \Delta \nu_{x}=-C a^{2} \text { and define } c=16 \pi C / A \\
& \text { then } d a / d N=(1 / 8) A a^{3} \sin \alpha \text { as before } \\
& \text { but } d \alpha / d N=(1 / 2) A a^{2}(\cos \alpha-C)+\delta_{\alpha} \\
& \text { and }\left(A / 4 \delta_{\alpha}\right) a^{4}(\cos \alpha-C)+a^{2}=\text { const. }
\end{aligned}
$$


When $c$ is greater than 1 , both the $\alpha=0$ and $\alpha=180^{\circ}$ fixed-points are at positive $\delta$, and there are none for negative $\delta$, however only $180^{\circ}$ has a separatrix. Figure 2 is drawn for $c=3$. The four fold "island" is relatively smaller for larger c. Because of the tune-shift, an adiabatic amplitude fills the inside at a $\delta / 2$ that is larger than before.

Further tuning towards $1 / 4$ moves some particles into the island, and they may be lost because the peak amplitude is too large. Particles of initially small amplitude - at tuning close to $1 / 4$ suffer the same relative increase and will be stable.

The island is not "filled" with beam, (in fact

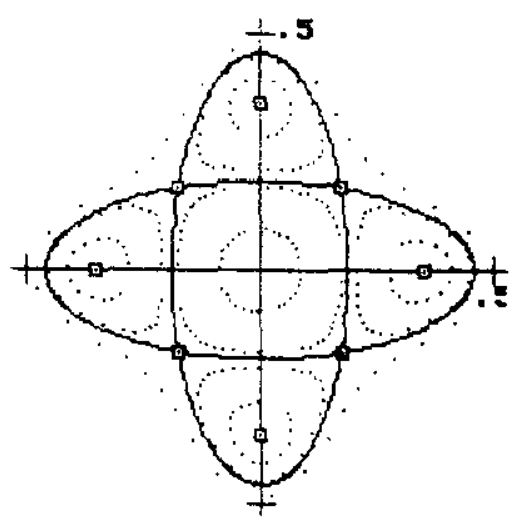

fig. 2 closed by $c=3$ for an infinitely slow tuning particles move directly to the outside), and the process of entering and leaving is complex. An initially uniform density disk, tuned thru a closed resonance, will have a distorted boundary and an effective phase dilution.

In general the effect of amplitude-dependent tune-shift on the low multipole resonances is to make them wide - particularly when we include the $b$ dependence - and effective only on larger amplitudes. High multipole resonances have much smaller driving terms, but see the same tune-shift and so have narrow closed islands which have little effect. This is why the beam is stable in spite of an apparently overwhelming number of resonances. As a pain-killer, however, tune-shift has bad side effects - see Distortion Functions - and cannot be used to alleviate poor magnet quality.

The significant strength parameter for a closed resonance is the area of the island compared to the area inside the separatrix. Some authors do express the strength in terms of "tune width", but there are no missing tunes and it is difficult to find a simple tuning width which is relevant. The particular example above is unique in that $A$ and $C$ have the same dimensions, and can be combined in a single amplitude dependence.

Some other solutions

Consider the 10-pole. It is no suprise that one will expand

$$
(\cos \varphi)^{5}=(1 / 16)(\cos 5 \varphi+5 \cos 3 \varphi+10 \cos \varphi),
$$

but it may be a suprise that the 10-pole will probably have $1 / 3$ 's resonances which are five times wider than its "principle" $1 / 5$ 's resonances! The sextupole resonances at the same tunes are probably larger, but not necessarily. 
One can find a solution for combinations of multipoles contributing to a single resonance, for example the constant of the motion at $1 / 3$ is

$$
\left(1 / 2 \delta_{a}\right)\left[\left(A_{3} a^{3}+(3 / 4) A 5 a^{5}+. .\right) \cos 3 \varphi-\left(B 3 a^{3}+(3 / 4) B s a^{5}+. .\right) \sin 3 \varphi\right]+a^{2}
$$

where the simple term $A a^{3}$ is now replaced by polynomials (one can eliminate $B_{3}$ by shifting, but that is all). As long as the polynomials are monotonic there is little change in the character of the resonance, but if they have roots, or multiple roots, then there are multiple sets of fixed-points and separatrices. If you, too, are addicted to recreational computation you will enjoy displaying some of these pretty resonance diagrams.

Another type of solution can be found for exactly superimposed resonances where one is a simple multiple of the other, for example in the octupole case when the tune is $2 / 4$ then both $4 \varphi$ and $2 \varphi$ in the expansion become resonant (with different driving terms). In this case the constant of the motion contains both angles. Again one finds some very interesting diagrams.

There is a process called feeddown which comes from a closed orbit displacement in the multipole element - from orbit distortion, momentum dis placement or physical misalignment of the element. Expanding $(X+d)=$ one finds $X^{\bullet}+m d X^{-1}$.. which creates feeddown to lower multipoles. (Vertical displacement in normal multipoles generates skews, and vice-versa.) For random multipoles these contributions can be large and explain the restricted momentum aperture and the emphasis on closed orbit control. For systematic elements feeddown caused by orbit distortion can produce unexpected huge effects, which I call semi-systematic resonances.

An orbit distortion may arise from a random set of dipole errors but the distortion itself is not random. The Fourier analysis of a random variable around the ring would probably have equal strength in all harmonics. The distorted orbit is dominated by one, or at most two harmonics at the nearest integers to the tune. A systematic array of multipoles will have its own harmonic structure at mutiples of the basic phase symmetry. Feeddown driving terms for $k-1$ resonances will be strong at the sums and differences of the orbit and multipole harmonics, which are normally terms that one would expect to be suppressed by the symmetry. Semi-systematic resonances within the normal tuning range must be avoided.

One sometimes hears speakers say that they have measured a resonance width, and using "so-and-so's" formula calculated an improbable multipole strength, implying that resonance theory is inadequate. Of course one cannot work backwards from tune to source multipole without many measurements; worse yet is to apply one-dimensional formulas to two-dimensional resonances. 


\section{Two-Dimensional Resonance}

The analysis of two-dimensional resonances is pure drudgery. Gone are the pretty diagrams, they are hidden in a four-dimensioned phase space. In fact, short of blowing-up the beam it is difficult to detect the resonance simultaneous plots of $x, x^{\prime}$ and $y, y^{\prime}$ are not helpful, just smeared - and the best one can offer is a simple plot of a vB $b$.

Each resonance, in its own way, defines "family" lines on an $a, b$ plot (hyperbolas or ellipses) and each particle is assigned to a particular family according to its values of $a$ and $b$, and remains on that line. For each family there is a single variable giving the position along the line and a "onedimensional" resonance complete with fixed-points and separatrices. Some family members are stable, some are not, and all considerations of tune-shift, polynomial driving terms and superimposed resonances apply. The arithmetic is obviously messy but the real drudgery is that each family is different and the problem must be solved many times over.

Nevertheless two-dimensional resosnances are more numerous and much stronger than their one-dimensional relatives. We will demonstrate this by devising and evaluating a suitable expression for the width of the principle resonances.

In the expression $m+n=k$, if $m$ and $k$ have the same parity then the resonance is from normal multipoles, $b_{k-1}$, otherwise it is from skew multipoles, ak-1. We illustrate the meaning of principle from the normal octupole

$$
B_{y}=-B 3 b_{3} X Y^{2} \text { and } B_{x}=B 3 b_{3} X^{2} Y
$$

which, as I am sure you can now easily see, involve the following

$$
\begin{aligned}
\Delta \varphi, \Delta \vartheta & \rightarrow \cos ^{2} \varphi \cos ^{2} \theta=(\cos 2 \varphi+2 \vartheta+\cos 2 \varphi-2 \theta+2 \cos 2 \varphi+2 \cos 2 \theta+2) / 8 \\
\Delta a & \rightarrow \sin \varphi \cos \varphi \cos ^{2} \theta=(\sin 2 \varphi+2 \vartheta+\sin 2 \varphi-2 \vartheta+2 \sin 2 \varphi) / 8 \\
\Delta b & \rightarrow \cos ^{2} \varphi \sin \vartheta \cos \vartheta=(\sin 2 \varphi+2 \vartheta-\sin 2 \varphi-2 \vartheta+2 \sin 2 \vartheta) / 8
\end{aligned}
$$

The principle resonance is the first sum term. The difference terms are a kind of non-linear coupling which is of practical importance for beam distortion $\left(\nu_{x}\right.$ near $\left.\nu_{y}\right)$, but in principle does not blow-up the beam. Notice that there is a contribution to the tune-shift polynomial, $a b^{2}$ term for $\nu_{x}$ and vice-versa, but we will ignore tune-shift. The remaining terms are presumed to be non-resonant at the principle tune. In other words, we are going to keep it simple. 
We now use the general expressions for the fields (for $b_{k-1}$ )

$$
\begin{aligned}
& B_{y}=B(-1)^{n / 2}\left(k_{m}\right)(m / k) b_{k-1} X^{m-1} Y^{n} \\
& B_{x}=-B(-1)^{a / 2}\left(k_{m}\right)(n / k) b_{k-1} X^{m} Y^{n-1}
\end{aligned}
$$

and, as before, we find $\Delta x, \Delta y$; then $\Delta a, \Delta \varphi, \Delta b, \Delta v$; and expand saving the first term, with the angle called $\alpha$. Again we separate starting angles from phase advance in the turn and collect a drivng term $A$ which is set to a positive maximum by the choice of observation point.

In this case we will express $a, b$ and everything else, except $A$ and $\sigma$, in units scaled by an ao which will collect the nuisance constants. In summary

$$
\begin{aligned}
& m \nu_{x}+n \nu_{y}=\text { integer } \div \delta / 2, \quad m+n=k, \quad \delta \text { perp. to res. line, } \\
& \alpha=m_{\varphi}+n \theta, \quad \delta_{\alpha}=\left(m^{2}+n^{2}\right)^{1 / 2} \pi \delta \\
& A=(-1)^{n / 2}\left\langle B \beta_{0} /\left[B_{0}\right]\right) \sum\left(h^{m} \mathrm{~V}^{n} b_{k-1}\right)_{s} \cos \alpha_{8} \quad \max \text {. } \\
& \left(a_{0}\right)^{k-2}=\left(2^{k-1} /\left(k_{m}\right)\right) \delta_{a} / A=\left(2^{k-1} /\left(k_{m}\right)\right)\left(m^{2}+n^{2}\right)^{y_{2}} \pi \delta / A \\
& d a / d N=\delta_{a}(m / k) a^{m-1} b^{n} \sin \alpha \\
& d b / d N=\delta_{a}(n / k) a^{m} b^{n-1} \sin \alpha \\
& d \alpha / d N=\delta_{\alpha}(1 / k)\left[\left(m^{2} a^{n-2} b^{n}+n^{2} a^{m} b^{n-2}\right) \cos \alpha+k\right] \\
& \text { I. } n a^{2}-m b^{2}=F \text { the " } F \text { " family line } \\
& \text { II. } \quad m^{2} a^{m-2} b^{n}+n^{2} a^{m} b^{n-2}=k \text { the fixed-roint line }\left(\alpha=180^{\circ}\right) \\
& \text { III. } \quad 2 a^{a} b^{2} \cos \alpha+a^{2}=\text { constant of motion. }
\end{aligned}
$$

Expression I comes from the combination of $\mathrm{d} a / \mathrm{dN}$ and $\mathrm{d} b / \mathrm{dN}$, and is an hyperbola. Every particle belongs to a family and, even if it blows-up, it cannot leave the family line. [For $2 \varphi-2 \theta$ the sign of $\mathrm{d} b / \mathrm{dN}$ is changed and $n a^{2}+m b^{2}=F$, which is an ellipse, so the amplitudes are bounded. It can be an elongated ellipse.]

The line of fixed-points in expression II has a wide variety of shapes for different resonances. The fixed-point amplitude pair, $a, b$, for a particular particle is the intersection with its family line. Substitution of these values and $\cos \alpha=-1$ in III gives the constant of motion for the family. One can then solve for the minimum amplitude pair using $\cos \alpha=0$, but one must use (I) to eliminate $a$ or $b$ first to remain on the family line.

In a similar manner one can find $\left(a^{2} b^{2}\right)$ averaze, over $\alpha$, which is related to the phase-space volume, and is the adiabatic "amplitude" far from resonance which just fills the separatrix at $\delta / 2$. The family relation must be maintained. Please see the example on page 16 . 
It is apparent that we must use some standard distribution of beam among the families in order to reduce the adiabatic line to a single measure of tuning width. I will use a gaussian beam with the same $\sigma$ in both planes. The amplitude distribution for one plane is a Rayleigh, or circular normal

$$
\begin{aligned}
P(a) & =\left(a / \sigma^{2}\right) \exp -a^{2} / 2 \sigma^{2} \text { da } \\
\text { or } \quad P(O \rightarrow a) & =1-\exp -a^{2} / 2 \sigma^{2} \quad \text { integrated }
\end{aligned}
$$

For two planes with the same $\sigma$ we have the folding of two circular normals

$$
\begin{aligned}
\mathrm{P}(a, b) & =\left(a b / \sigma^{4}\right) \exp -\left(a^{2}+b^{2}\right) / 2 \sigma^{2} d a d b \\
\text { or } \quad \mathrm{P}\left(a^{2}, b^{2}\right) & =\left(1 / 4 \sigma^{2}\right) \exp -\left(a^{2}+b^{2}\right) / 2 \sigma^{2} d\left(a^{2}\right) d\left(b^{2}\right)
\end{aligned}
$$

If one writes $r^{2}=a^{2}+b^{2}$ then the cummulative distribution

$$
\mathrm{P}(0 \rightarrow \mathrm{r})=1-\left(1+\mathrm{r}^{2} / 2 \sigma^{2}\right) \cdot \exp -\mathrm{r}^{2} / 2 \sigma^{2}
$$

For our scaled values we must replace $\sigma$ in the above by $\sigma / a$.

I define the tuning width as the difference between $10 \%$ loss points when tuning perpendicular to the resonance. To find this width one first finds the adiabatic amplitudes for many values of $F$, then guesses a value for $\sigma / a_{0}$ and numerically integrates the distribution function to find how much of the beam is below the adiabatic line (expressed as $\left.a^{2}, b^{2}\right)$, and adjusts $\sigma / a_{0}$ until the value is $90 \%$.

$$
\begin{aligned}
\text { Let } & {\left[\sigma / a_{o}\right] .9=\rho, \text { and } \delta=W \sigma^{k-2} A / \pi } \\
\text { intrinsic width } & W=\left(1 / \rho^{k-2}\right)\left(1 / 2^{k-1}\right)\left(k_{m}\right) /\left(m^{2}+n^{2}\right)^{1 / 2} .
\end{aligned}
$$

This calculation is illustrated by the lower diagram for the example on page 16, where I have superimposed the final cummulative $r^{2}$ distribution. You can see which families cause most of the loss for this particular resonance.

On the next page there is a summary table of W's, which shows that resonances near $m=n$ are much the most important. The primary factor is the binomial coefficient $\left(k_{m}\right)$ from the multipole expansion.

The comparison of tuning widths between different values of $k$ involves the relative magnitude of multipoles. For random errors in the Doubler, and for a beam with $\sigma=1 \mathrm{~cm}$. (large!), one can expect A $\sigma^{\mathrm{k}-2}$ to probably fall by a factor of 15 for two steps in $k$. For $m=n, W$ rises by 3 for two steps, so the probable widths fall fairly rapidly with increasing $k$. For systematic errors there is no prediction.

The comparison of tuning widths for different $m, n$ with the same $k$ involves only $W$ and $h^{\omega} V^{n}=\left(\beta_{x}{ }^{m / 2} \beta_{y}^{n / 2} / \beta_{0}\right)$. For multipole elements installed near quadrupoles, the latter term is 1 for one-dimensional resonances and 
$(.76)^{k}\left(60^{\circ}\right.$ cells $)$ or $(.64)^{k}\left(90^{\circ}\right.$ cells $)$ for resonances with $m=n=k / 2$. This somewhat mitigates the rise in $W$ but it introduces a dependence on the choice of the phase advance for the cell.

For random errors one must use the rms. average over the cell. This has been combined with $W$ in the curves on page 18. Each curve is normalized to the probable width for the one-dimensional resonances. The original purpose of these curves was to convince designers that high phase advance per cell, which costs very little extra, substantially reduces resonance problems. They do show that two-dimensional resonances are dominant.

\section{Final Comments}

One can never expect to measure widths as given above. As we have seen real resonances are combinations and include tune shifts, polynomial driving terms and overlapped resonances. This does not invalidate the basic conclusions because the causes - binomial coeficients and $\beta$ dependence - are so fundamental. It is true that the quantitative relation between resonances and good accelerator performance is not obvious, and this led me to nonresonant Distortion Functions and to the "smearing" criterion which reaches the same general conclusion quantitatively. Nevertheless there are a number of reasons why one should be very familiar with the theory of resonances.

Resonances large enough to be annoying in a ring with an adequately low distortion are most probably from some overlooked systematic multipole effect, particularly if they appear during low-beta turn-on which destroys phase symmetry. Except that the resonance is probably two-dimensional, there is no probability theory for goofs and one must understand all the simple and subtle ways that could produce the particular resonant frequency. The converse applies to the goof-free design of new multipole elements for the ring.

The design of intentional resonances is the real fun. Slow extraction uses a designed resonance but there must be many more applications, particularly in a rational ring like the Doubler and with today's superconducting higher multipoles. As an example consider a "separatrix" beam scraper to remove large betatron amplitudes. A strong amplitude dependence will suppress momentum problems - so how about 10-poles, used at $1 / 3$ 's for increased strength, and in two-dimensions because that's what we should scrape, and maybe a little non-linear coupling, and perhaps we should tailor the tune-shift function, and ... but don't let me design it - you design it. 
EXAMPLE. $\quad 2 \nu_{x}+3 \nu_{y}=$ unteger $+\sqrt{13} \delta / 2$

$A=\frac{j_{0} B_{0}}{(B p)} ; a_{4}\left(n^{2} v^{3}\right) \cos \alpha_{3} d l$.

$$
\alpha=2 \varphi+3 \vartheta \text {. }
$$

scale ampis bu $a_{0} . a_{0}^{3}=\frac{16 \sqrt{13}}{10} \delta \frac{\pi}{A}$

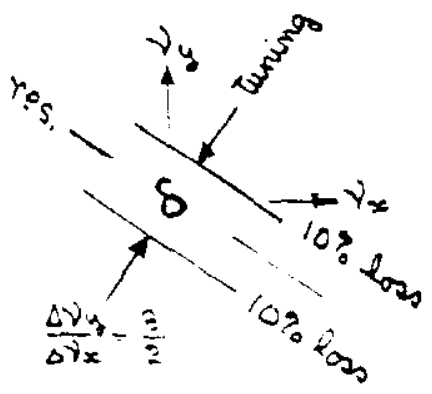

motion constracneo to

$F=3 a^{2}-2 b^{2}$.

Fixed pis. $4 b^{3}+9 a^{23} z=5$ use $2 a^{2} b^{3} \cos \alpha_{0}+a^{2}+b^{2}=$ conot.

to follow just stabie priticle aliong $F$, firm $x_{0}=$ iEC 'Fixespt: $\operatorname{to} x_{0}=0$. Fund average coect $\left.x\right) \quad b$ $3^{2} b^{2}$ which guti phase space Niums.

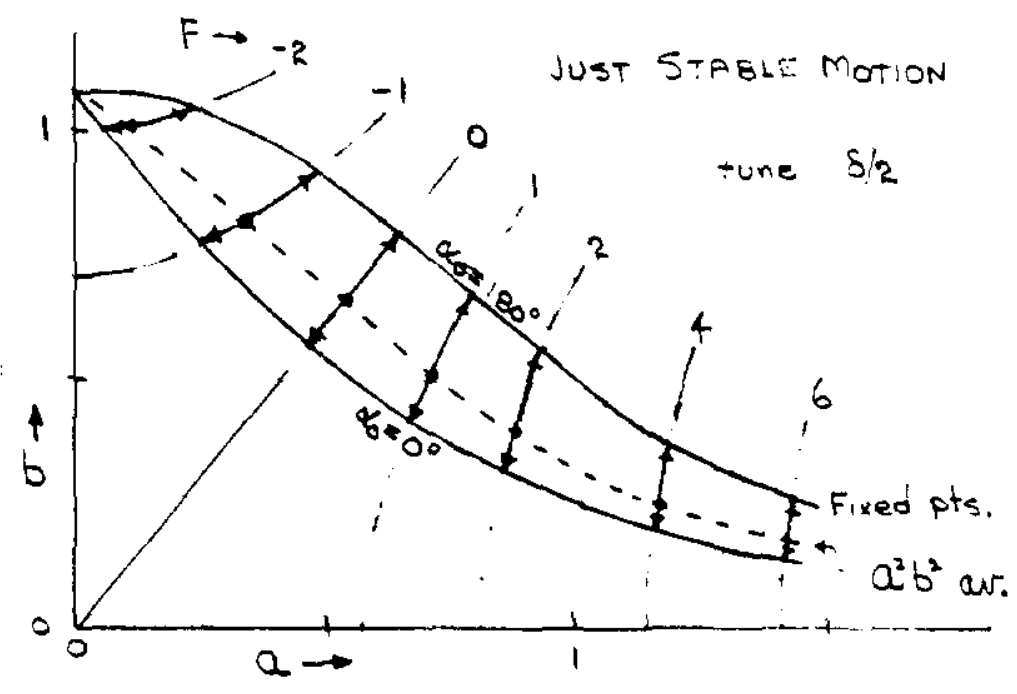

PDiust $\sigma$ of

GRUSSIR.N Ber.

(sume iner a revt)

$\leq 0 \quad 10 \%$ is bejond

Amid. Litait.

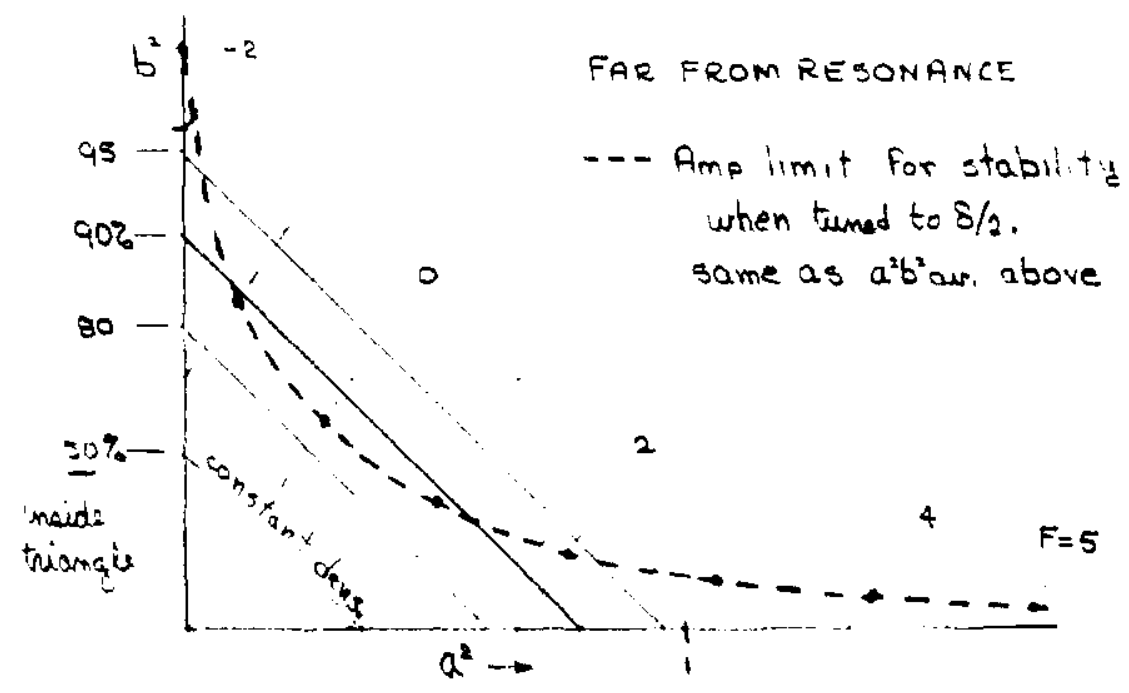

$\sigma / a_{0}=.318 \rightarrow \delta=5.38 A \sigma^{3} / \pi$ 
INTFINSIS WIOTH $W$ FOR RESONANCES $m V_{x}+n V_{y}$

$$
\text { in } 10^{2} \text { loss. } \quad k=m+n
$$

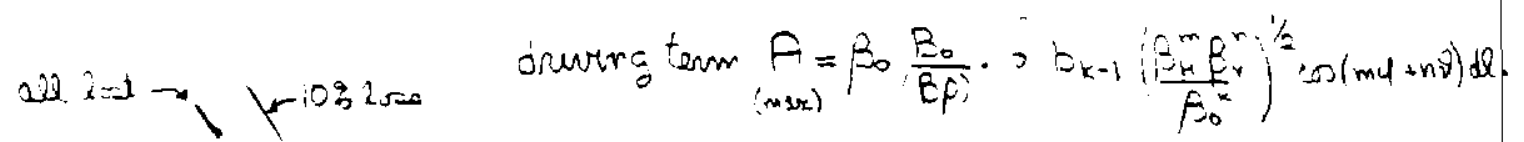

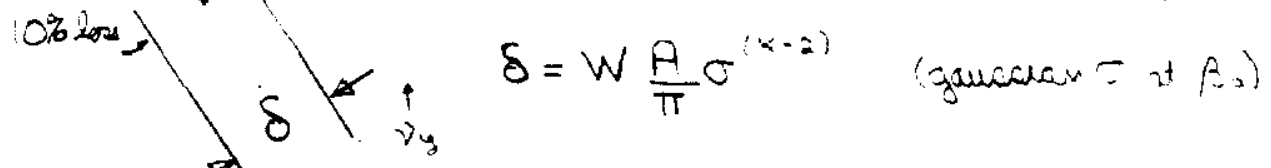
$\rightarrow x_{\sin } \rightarrow \nu_{x}$

$k=3 i \quad 4 \quad 5$

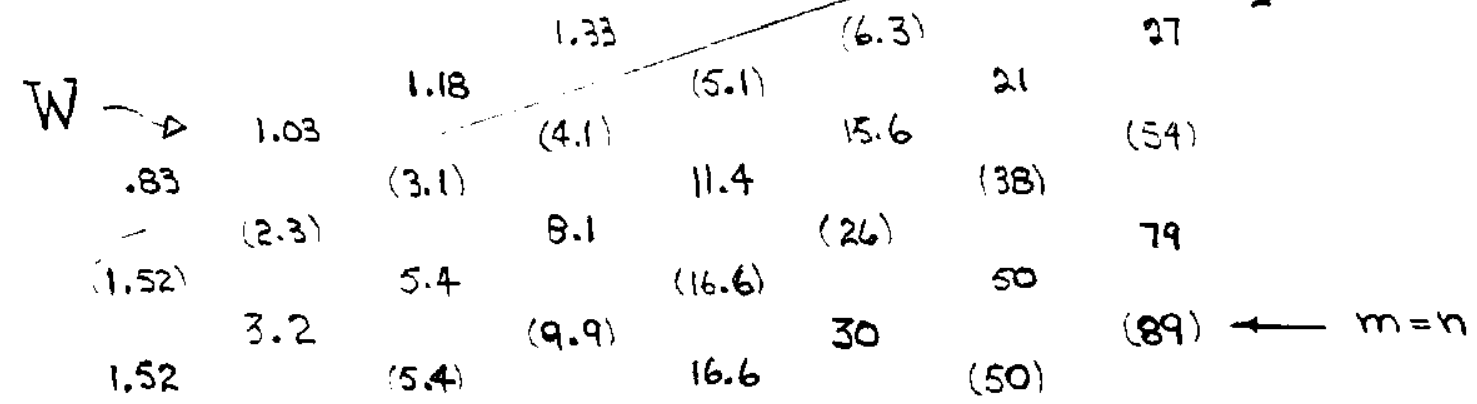
$\begin{array}{llll}2.3) & 8.1 & 26 & 79\end{array}$ $(.83) \int_{1.03^{5}}^{3.1}(4.1)^{(11.4)} \quad 15.6 \quad 38$

"skew" issonalises

is $I_{0-1}$ is $A$.
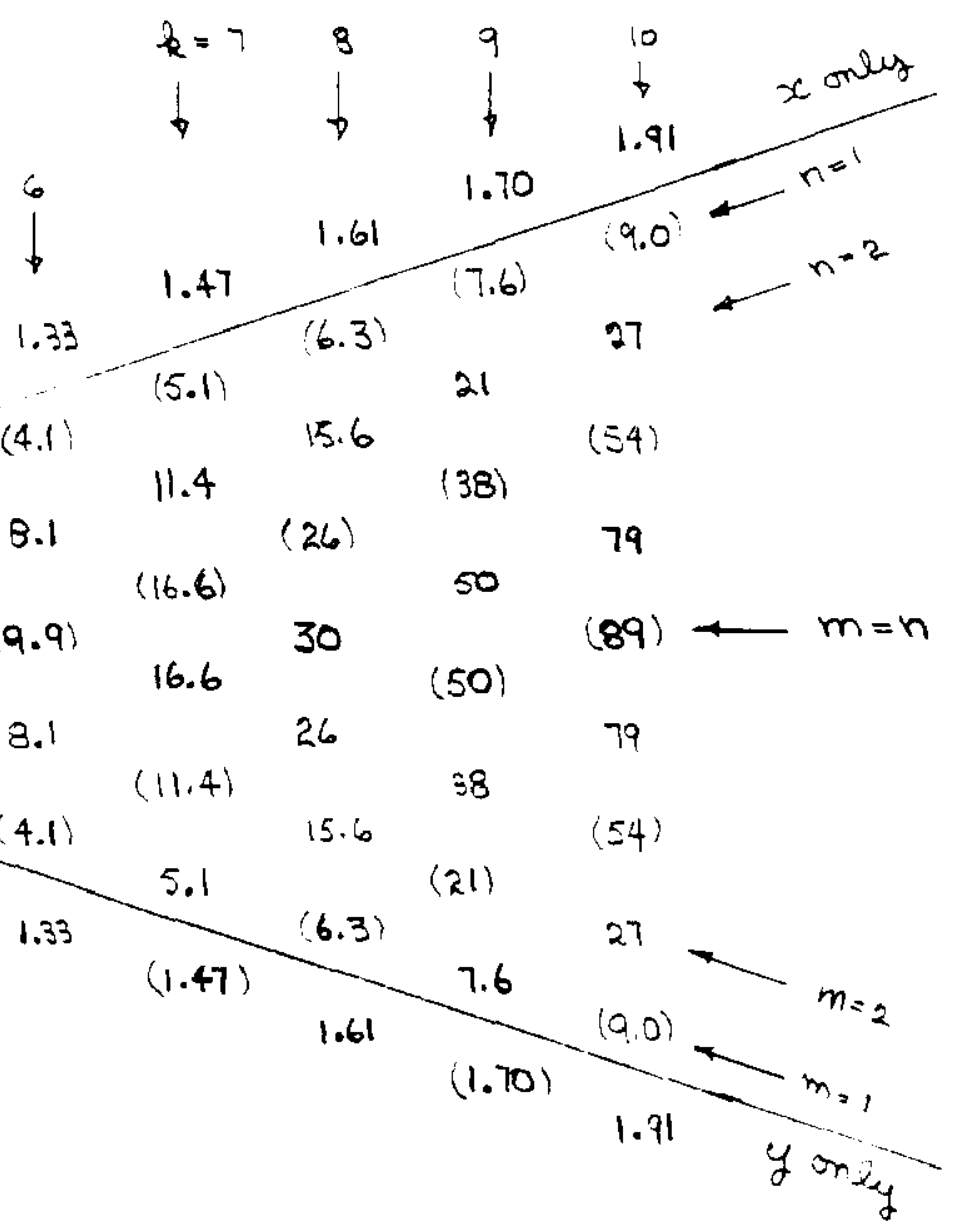

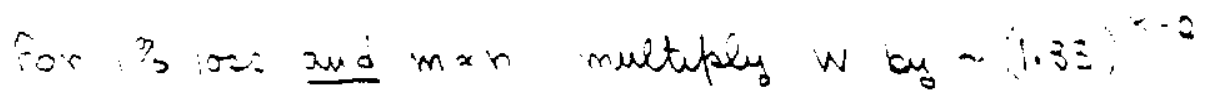

$$
\begin{array}{ll}
k=t & 1.75 x \\
k=-1 & 4.2 x \\
k=10 & 10 x
\end{array}
$$


Resonance Widths no. Phase advance/Cell.

Fon each deagram widthe are relature to 1 fer one-demensear at $30^{\circ}$ Randow enrors fo and emettanes sse rastant

The incread $\rightarrow m=n$ co intrumaic in nexonance numerokcy.

The decrease with $\varphi$ is from $\beta_{\mu}^{\pi / x} \beta_{r}^{x / 2}$ in
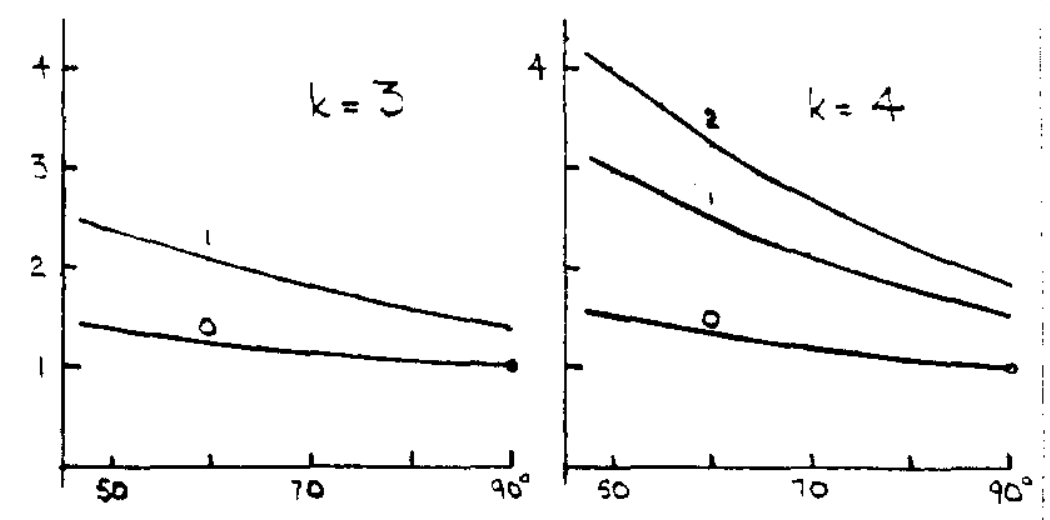
the driveng term.
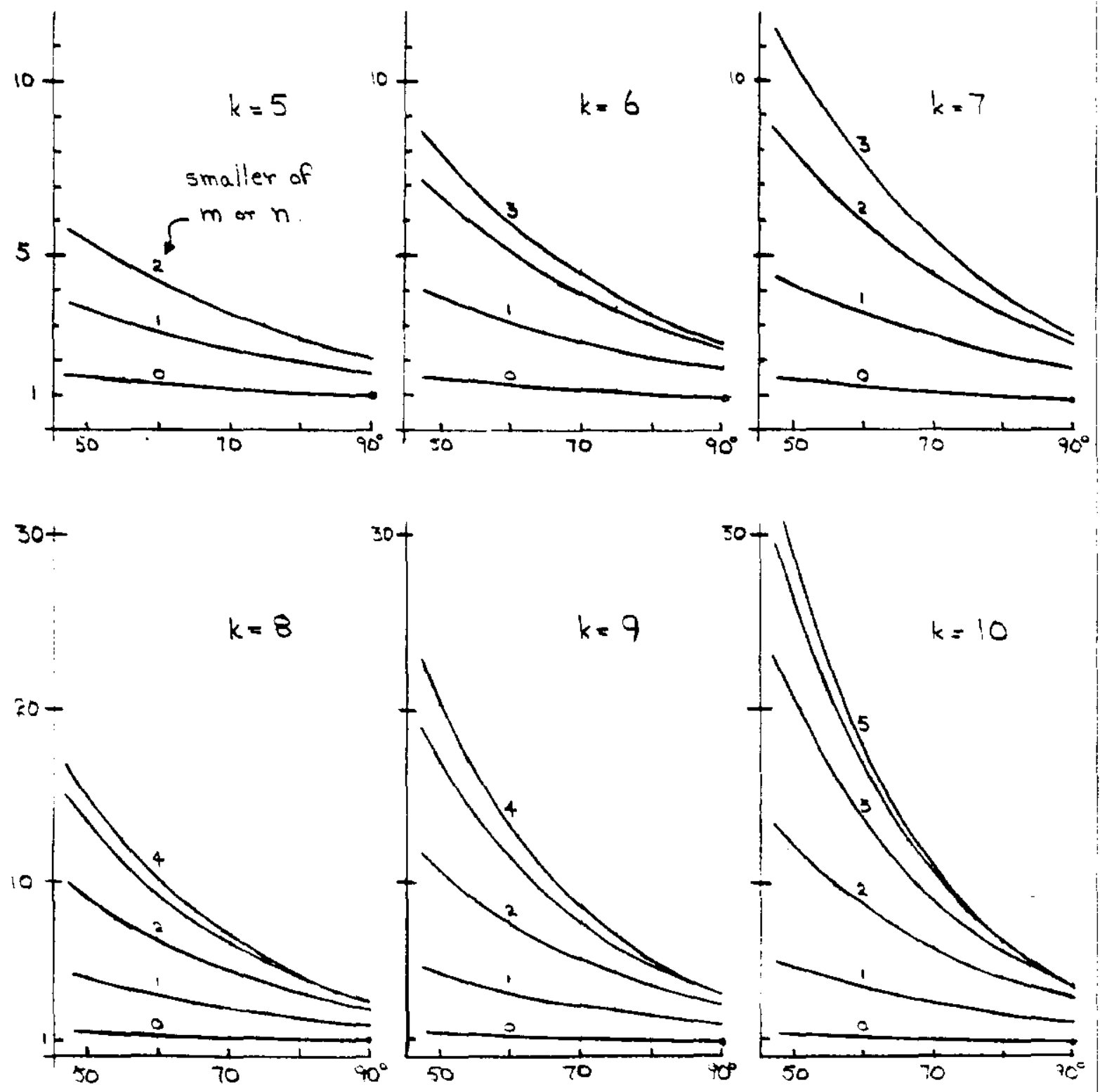